\title{
Rare Case of Nodular-type Muscle Sarcoidosis with Typical 3-Stripes Sign
}

QUN ZHU, MM, West China Medical School of Sichuan University; GENG YIN, MD; GANG LIU, MD, Department of Rheumatology and Immunology, West China Hospital of Sichuan University, Chengdu, Sichuan, China. Dr. G. Yin is co-first author. Address correspondence to Dr. G. Liu, 37 Guoxue Alley, Chengdu, Sichuan, China, 610041. E-mail: huaxi_liugang@163.com. J Rheumatol 2015;42:897-8; doi:10.3899/jrheum.141186

Sarcoidosis is a multisystem noncaseating granulomatous disorder that seldom affects the muscle ${ }^{1}$. It is rare for nodular muscle sarcoidosis to be the initial and sole manifestation and this may present a diagnostic dilemma for clinicians.

A 59-year-old previously healthy woman visited our rheumatology clinic complaining of masses in her calves for 6 months with swelling when standing on her feet. High-resolution computed tomography of the chest demonstrated that bilateral lungs and mediastinum were normal. Contrast-enhanced magnetic resonance imaging (MRI) of the calves revealed multifocal abnormal signals: on axial T1- and T2-weighted images the nodules showed a central, star-shaped structure with low signal intensity. The peripheral area showed high signal intensity on T1-weighted images and markedly high signal intensity on T2-weighted images (Figure 1). On coronal MR images, the nodules showed a typical 3-stripes sign: an inner stripe of low signal intensity with outer stripes of marked high intensity (Figure $2)$. Muscle biopsy revealed noncaseating granulomas. Laboratory tests were normal and other granulomatous disorders, such as tuberculosis and deep fungal infections, were ruled out on histology and culture of the lesions. Therefore the final diagnosis was nodular type muscle sarcoidosis, and the patient received treatment with methotrexate $10 \mathrm{mg}$ once a week and gradually tapering doses of oral prednisolone for 3 months. Swelling of the calves disappeared gradually during followup.

Muscle involvement is uncommon in sarcoidosis and palpable nodular-type muscle sarcoidosis is very $\operatorname{rare}^{2,3}$. When a mass exhibits the typical 3-stripes sign on MRI, nodular muscle sarcoidosis should be the first consideration for rheumatologists.

\section{REFERENCES}

1. Vardhanabhuti V, Venkatanarasimha N, Bhatnagar G, Maviki M, Iyengar S, Adams WM. Extra-pulmonary manifestations of sarcoidosis. Clin Radiol 2012;67:263-76.

2. Yamamoto T, Nagira K, Akisue T, Marui T, Hitora T, Nakatani T, et al. Aspiration biopsy of nodular sarcoidosis of the muscle. Diagn Cytopathol 2002;26:109-12.

3. Suresh S, Tirabosco R, Saifuddin A, O'Donnell P. An unusual presentation of muscular sarcoidosis. Skeletal Radiol 2007;36:995-8.

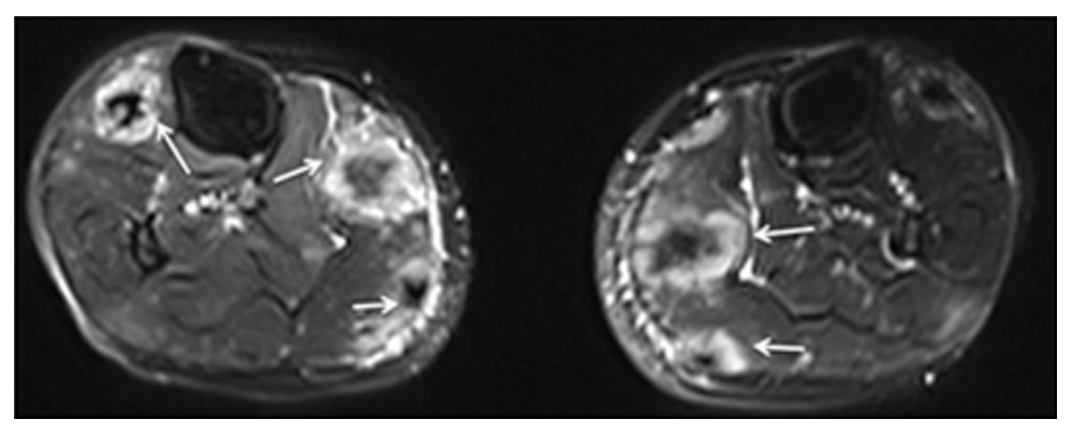

Figure 1. Axial fat-suppressed T2-weighted image of the calves shows nodules with a central, star-shaped low signal intensity structure and peripheral marked high intensity (arrows). 


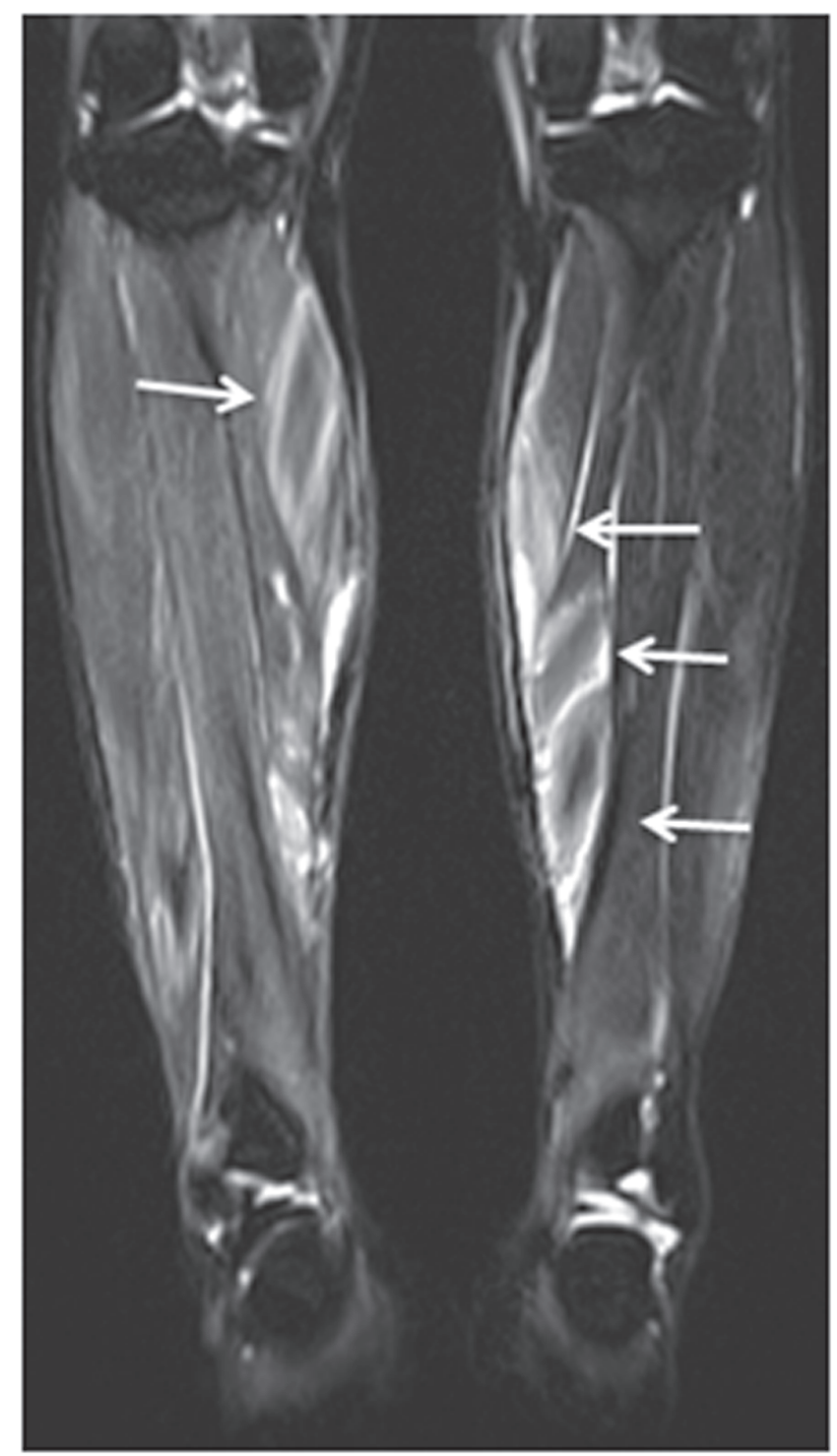

Figure 2. Coronal fat-suppressed T2-weighted image shows the multifocal intramuscular nodules as an inner stripe of decreased signal intensity with outer stripes of increased signal intensity (arrows), the so-called 3-stripes sign. 